\title{
The influence of surface treatment on hydrophobicity, protein adsorption and microbial colonisation of silicone hydrogel contact lenses
}

\author{
Lívia Santos ${ }^{\mathrm{a}}$, Diana Rodrigues ${ }^{\mathrm{a}}$, Madalena Lira ${ }^{\mathrm{b}}$, M. Elisabete C.D. Real Oliveira ${ }^{\mathrm{b}}$, \\ Rosário Oliveira ${ }^{\mathrm{a}}$, Eva Yebra-Pimentel Vilar ${ }^{\mathrm{c}}$, Joana Azeredo ${ }^{\mathrm{a}, *}$ \\ ${ }^{a}$ IBB-Institute for Biotechnology and Bioengineering, Centre for Biological Engineering, University of Minho, \\ Campus de Gualtar 4710-057 Braga, Portugal \\ ${ }^{\mathrm{b}}$ Centre of Physics (Optometry), University of Minho, Braga, Portugal \\ ${ }^{\mathrm{c}}$ University of Santiago de Compostela, Santiago de Compostela, Spain
}

\begin{abstract}
Purpose: To evaluate the influence of surface treatment of silicone-hydrogel CL on lens hydrophobicity, protein adsorption and microbial colonisation by studying several silicone hydrogel contact lenses (CL) with and without surface treatment. The lenses used in this study were Balafilcon A, Lotrafilcon A, Lotrafilcon B and Galyfilcon A. A conventional hydrogel CL (Etafilcon A) was also tested.

Methods: Hydrophobicity was determined through contact angle measurement using the advancing type technique on air. The type and quantity of proteins adsorbed were assessed through SDS-PAGE and fluorescence spectroscopy, respectively. Microbial colonisation was studied by removing the microbes from the lenses through sonication, and counting the colony-forming units on agar plates.

Results: Regarding hydrophobicity, both surface and non-surface-treated silicone hydrogel CL were found to be hydrophobic, and the conventional hydrogel CL was found to be hydrophilic. Concerning protein adsorption, different protein profiles were observed on the several lenses tested. Nevertheless, the presence of proteins with the same molecular weight as lysozyme and lactoferrin was common to all lenses, which is probably related to their abundance in tears. In terms of total protein adsorption, silicone hydrogel CL did not exhibit any differences between themselves. However, the conventional hydrogel Etafilcon A adsorbed a larger amount of proteins. Regarding microbial colonisation, Balafilcon A exhibited the greatest amount of colonising microbes, which can be due to its superior hydrophobicity and higher electron acceptor capacity.

Conclusion: This study suggests that silicone hydrogel lenses adsorb a lower amount of proteins than the conventional hydrogel lenses and that this phenomenon is independent of the presence of surface treatment. Concerning microbial colonisation, the surface treated Balafilcon A, exhibited a greater propensity, a fact that may compromise the lens wearer's ocular health.
\end{abstract}

(C) 2007 British Contact Lens Association. Published by Elsevier Ltd. All rights reserved.

Keywords: Silicone hydrogel contact lenses; Surface treatment; Protein adsorption; Microbial colonisation

\section{Introduction}

Silicone hydrogel contact lenses (CL) are the latest kind of soft lenses commercially available. This type of lens provides excellent oxygen transmissibility to the cornea on account of silicone's high oxygen transmissibility when compared to the conventional hydrogel CL $[1,2]$. Silicone is a hydrophobic polymer, and for this reason most of the silicone-based CL possesses surface treatment, which

\footnotetext{
* Corresponding author. Tel.: +351 253604419 .

E-mail address: jazeredo@deb.uminho.pt (J. Azeredo).
}

decreases the surface hydrophobicity. The reduction in hydrophobicity gives a greater comfort to the wearer and additionally prevents the formation of deposits such as lipids and proteins, as well as microbial colonisation [3-6]. The reduction in the lens surface hydrophobicity can be obtained through two methods. The first one consists in performing a treatment on the lens surface, which can be achieved in a gas plasma reactive chamber by creating an ultra-thin permanent coating in the cases of Lotrafilcon A and Lotrafilcon B (Ciba Vision), or by plasma oxidation, transforming the silicone into silicate compounds, in the case of Balafilcon A (Bausch \& Lomb, Inc.) (Table 1). The second method consists in the 
Table 1

Contact lenses properties

\begin{tabular}{|c|c|c|c|c|c|}
\hline Commercial name & Manufacturer & Material & FDA group & Water content & Surface treatment \\
\hline Acuvue $^{\circledR}$ & Johnson \& Johnson Vision Care & Etafilcon A & IV & $58 \%$ & No \\
\hline Acuvue $^{\circledR}$ Advance $^{\mathrm{TM}}$ & Johnson \& Johnson Vision Care & Galyfilcon & I & $47 \%$ & No \\
\hline Purevision $^{\mathrm{TM}}$ & Bausch \& Lomb, Inc. & Balafilcon A & III & $36 \%$ & Plasma oxidation \\
\hline Focus ${ }^{\circledR}$ Night \& Day $^{\mathrm{TM}}$ & CIBA Vision & Lotrafilcon A & I & $24 \%$ & $25 \mathrm{~nm}$ plasma \\
\hline $\mathrm{O}_{2} \mathrm{Optix}^{\mathrm{TM}}$ & CIBA Vision & Lotrafilcon B & I & $33 \%$ & $25 \mathrm{~nm}$ plasma \\
\hline
\end{tabular}

incorporation of a wetting agent such as polyvinyl pyrrolidone (PVP), which is the case of Galyfilcon A (Table 1) (Johnson \& Johnson Vision Care).

Silicone hydrogel CL, despite the advantages they offer due to their high oxygen transmissibility, also present some pitfalls, which are related to the migration of the silicone hydrophobic moieties to the lens surface [7]. As mentioned above, less hydrophobic surfaces are advantageous, since they prevent protein adsorption and microbial colonisation.

The main goal of this study is to evaluate the influence of surface treatment on CL hydrophobicity, protein adsorption, and microbial colonisation, since lenses with surface treatment are expected to exhibit different behaviours than non-surface-treated lenses. For that, a group of human volunteers wore four types of silicone hydrogel lenses: three surface-treated CL and one non-surface-treated CL. A conventional hydrogel CL (Etafilcon A, Johnson \& Johnson Vision Care) was also tested. The implications of protein adsorption and microbial adhesion have already been established through "in vitro" studies [8-13]. However, due to the complexity of the ocular environment, it is difficult to mimic all the conditions affecting protein adsorption and microbial adhesion. Therefore, "in vivo" experiments offer potentially more reliable and conclusive results. Moreover, the degree of protein adsorption and microbial colonisation in Lotrafilcon $B$ has never been reported before.

\section{Materials and methods}

\subsection{Contact lenses and subjects}

The properties of the CL used in this study are detailed in Table 1 . The experiments were performed on CL previously worn by a group of 31 subjects from both sexes. The group of subjects was selected according to several criteria: the subjects had never worn CL before, were not taking any medication during the trial, did not suffer from any kind of ocular allergy, and had no predisposition to dry eye syndrome. Each type of silicone hydrogel CL was worn by approximately 8 subjects and the conventional hydrogel CL was worn by all the 31 patients, since each individual wore a certain type of silicone hydrogel $\mathrm{CL}$ in one eye and the conventional hydrogel CL in the other one. The subjects were instructed to wear their lenses on a daily wear schedule for 12 and $14 \mathrm{~h}$ and to clean and disinfect the CL overnight with a multipurpose lens care solution (ReNu MultiPlus ${ }^{\circledR}$, Bausch \& Lomb, Inc.). The patients were not informed about the brand or type of lenses they were using. Silicone hydrogel CL were replaced every 30 days and the conventional hydrogel CL were replaced every 15 days during the 6-month period. At the end of each wearing period, the $\mathrm{CL}$ were collected, placed in sterile saline solutions, and preserved at $4{ }^{\circ} \mathrm{C}$ until further analysis.

According to the manufacturer's instructions, Galyfilcon A should only be worn for a 15-day period. However, in this study, patients wore this type of lens for 30 days, in order to make possible the comparison with the other silicone hydrogel CL tested.

It must be stressed that full ethics approval was obtained, and clinical cover was provided during the trials.

\subsection{Culture medium and solutions}

The culture medium used in this work was tryptic soy agar (Merck, Germany). This medium was prepared according to the manufacturer's instructions, sterilised and transferred to Petri dishes. A solution of $\mathrm{NaCl}(0.9 \%$, Sigma-Aldrich, Germany) was also prepared and sterilised.

\subsection{Contact angle measurements}

CL hydrophobicity was determined through the measurement of the advancing contact angle on air with a measurement apparatus. The measurements were performed at room temperature using three standard liquids of different polarities-Millipore water, formamide and 1-bromonaphtalene. Water and formamide are polar liquids, whereas 1bromonaphtalene is non-polar. For the measurements, unworn CL were removed from their original blisters and cut into quarters. Each quarter was flattened onto a microscope slide and the excess water was gently removed with a tissue paper. The measurements were immediately performed, in order to avoid lens dehydration, using the apparatus OCA 20 (DataPhysics, Germany). For each standard liquid, $4 \mathrm{CL}$ from each type were tested and 4 measurements per lens were performed.

\subsection{Polyacrylamide gel electrophoresis (SDS-PAGE)}

The types of proteins adsorbed onto worn CL were determined through SDS-PAGE, with a 10\% gradient gel. 
For protein extraction, lenses were cut into quarters and incubated in $100 \mu \mathrm{l}$ of electrophoresis buffer $(1 \mathrm{mM}$ EDTA, $10 \mathrm{mM}$ Tris-HCl pH 8.0, 2.5\% SDS and 5\% $\beta$ mercaptoethanol). After boiling for $15 \mathrm{~min}$, the CL were centrifuged for $10 \mathrm{~min}$ at $9000 \mathrm{rpm}$. The supernatant was removed and applied to a $10 \%$ gradient gel. Electrophoresis was performed in the MINI-PROTEAN ${ }^{\circledR} 3$ Cell (BIORAD, USA) using $60 \mathrm{~V}$. Gels were stained with silver nitrate.

\subsection{Fluorescence spectroscopy}

The total amount of proteins adsorbed onto worn CL was estimated by fluorescence spectroscopy. When excited at $280 \mathrm{~nm}$, proteins emit fluorescence due to the presence of fluorophore tryptophan [14]. Since there is no standard solution of tear-film proteins, the method was calibrated with different concentrations of bovine serum albumin (BSA) (Sigma-Aldrich, Germany). An excellent linearity between the BSA concentration and fluorescence intensity was found $(Y=1.895 x+6.6409 ; R=0.9998)$. Protein extraction was performed as described by Keith et al. [15]. According to the authors' procedures, CL were soaked in extraction buffer (acetonitrile and $0.2 \%$ trifluoroacetic acid (50:50)) and incubated in an orbital shaker (140 rpm) overnight. After this period, lenses were centrifuged for $10 \mathrm{~min}$ at $9000 \mathrm{rpm}$. Samples were analysed at an excitation wavelength of $280 \mathrm{~nm}$ and an emission of $360 \mathrm{~nm}$ (Spectrofluorimeter Jasco FP-6200, Japan). The measurements were performed in a quartz cell (Hellma, Germany).

\subsection{Colony-forming units}

Microbial colonisation was evaluated through the enumeration of colony-forming units (CFU). After wear, each CL was aseptically removed from the eye of the volunteer and placed in $1 \mathrm{ml}$ of sterile saline solution $(0.9 \%$ $\mathrm{NaCl}$ ). The lenses were sonicated (450W Ultrasonic Processor, Cole \& Parmer, USA) for $1 \mathrm{~min}$ at an amplitude of 20 with a (1/8) in. probe. The suspension was spread onto a TSA plate and the CFU were enumerated after 24 and $48 \mathrm{~h}$ of incubation at $37{ }^{\circ} \mathrm{C}$. The sonication time and power were optimised in order to detach the maximum number of adhered cells without cell disruption (assessed by plating the final suspension onto TSA plates).

\subsection{Statistical analysis}

The total amounts of proteins adsorbed onto the different types of CL were compared through one-way ANOVA, and the amounts of microbial cells colonising each CL were compared using the non-parametric Mann-Whitney $U$-test at a $95 \%$ confidence level. The statistical analysis was performed using the statistical program SPSS (Statistical Package for the Social Sciences).

\section{Results}

\subsection{Hydrophobicity}

Hydrophobicity was calculated as the free energy of interaction between two identical surfaces (s) immersed in water (w) [16]:

$$
\begin{aligned}
\Delta G_{\mathrm{sws}}^{\mathrm{tot}}= & -2\left(\sqrt{\gamma_{\mathrm{s}}^{\mathrm{LW}}}-\sqrt{\gamma_{\mathrm{w}}^{\mathrm{LW}}}\right)^{2} \\
& -4\left(\sqrt{\gamma_{\mathrm{s}}^{+} \gamma_{\mathrm{s}}^{-}}+\sqrt{\gamma_{\mathrm{w}}^{+} \gamma_{\mathrm{w}}^{-}}-\sqrt{\gamma_{\mathrm{s}}^{+} \gamma_{\mathrm{w}}^{-}}-\sqrt{\gamma_{\mathrm{s}}^{-} \gamma_{\mathrm{w}}^{+}}\right)
\end{aligned}
$$

$\Delta G_{\mathrm{sws}}^{\mathrm{tot}}$ expresses the degree to which the attraction of the surface (s) towards water (w) is greater (hydrophilicity) or smaller (hydrophobicity) than the attraction between two moieties of that surface. Thus, when the global free energy of interaction between two identical surfaces (s) immersed in water is repulsive (has a positive value) the surface (s) is considered hydrophilic. On the other hand, the more negative $\Delta G_{\text {sws }}^{\text {tot }}$ is, the higher the solid surface hydrophobicity is.

The values of the surface tension components and hydrophobicity are detailed in Table 2. From the results obtained it can be concluded that silicone hydrogel CL are hydrophobic, since $\Delta G_{\mathrm{sws}}^{\mathrm{tot}}$ is $<0$, and the conventional hydrogel CL is hydrophilic. Balafilcon A's surface presents the greatest surface hydrophobicity and can be considered a great electron acceptor (high value of $\gamma^{+}$).

\subsection{Types of proteins adsorbed}

The molecular weights of the adsorbed proteins are detailed in Table 3. According to the results obtained, every lens material exhibited a specific protein profile, Galyfilcon A being the lens presenting a greater variety of molecular weights. Proteins with molecular weights similar to those of lactoferrin and lysozyme were the most frequently found in the lenses tested.

\subsection{Total amount of proteins}

The estimated amounts of proteins adsorbed are present in Table 4. It is possible to conclude that all silicone hydrogel CL exhibit lower levels of protein adsorption $(p=0.000)$

Table 2

Apolar component $\left(\gamma^{\mathrm{LW}}\right)$, electron donator $\left(\gamma^{-}\right)$and electron acceptor parameter $\left(\gamma^{+}\right)$of the lens surface tension and hydrophobicity expressed in $\mathrm{mJ} / \mathrm{m}^{2}$

\begin{tabular}{lrrrr}
\hline Material & \multicolumn{1}{c}{$\gamma^{\mathrm{LW}}$} & \multicolumn{1}{c}{$\gamma^{+}$} & \multicolumn{1}{c}{$\gamma^{-}$} & \multicolumn{1}{c}{$\Delta G_{\mathrm{sws}}^{\text {tot }}$} \\
\hline Etafilcon A & 28.34 & 0.83 & 7.40 & 23.14 \\
Galyfilcon & 3.59 & 1.63 & 13.50 & -36.17 \\
Balafilcon A & 5.53 & 11.50 & 7.41 & -39.40 \\
Lotrafilcon A & 39.40 & 2.16 & 12.37 & -27.10 \\
Lotrafilcon B & 35.60 & 3.00 & 7.40 & -34.24 \\
\hline
\end{tabular}

Surface tension components and lens surface hydrophobicity $\left(\Delta G_{\mathrm{sws}}^{\mathrm{tot}}\right)$. 
Table 3

Proteins of different molecular weights adsorbed onto different worn contact lenses (\%)

\begin{tabular}{|c|c|c|c|c|c|}
\hline Adsorbed proteins (Mw, kDa) & Etafilcon A & Galyfilcon A & Balafilcon A & Lotrafilon A & $\overline{\text { Lotrafilcon B }}$ \\
\hline 14.4 (lysozyme) & 29.27 & 5.70 & 11.76 & 14.28 & 0.00 \\
\hline 21.0 (lipocalin) & 7.31 & 5.70 & 0.00 & 0.00 & 25.00 \\
\hline 80.0 (lactoferrin) & 12.20 & 17.14 & 11.76 & 14.28 & 50.00 \\
\hline 37.0 (igA) & 4.88 & 8.60 & 5.88 & 0.00 & 0.00 \\
\hline 66.2 (human serum albumin) & 4.88 & 11.40 & 0.00 & 14.28 & 0.00 \\
\hline Other proteins & 14.46 & 51.56 & 70.60 & 57.16 & 25.00 \\
\hline
\end{tabular}

Table 4

Fluorescence intensity at $360 \mathrm{~nm}$ of the contact lens extract

\begin{tabular}{lc}
\hline Etafilcon A & $472.97 \pm 196.95^{*}$ \\
Galyfilcon A & $9.19 \pm 5.53$ \\
Balafilcon A & $44.64 \pm 15.24$ \\
Lotrafilcon A & $20.45 \pm 10.85$ \\
Lotrafilcon B & $35.34 \pm 31.96$
\end{tabular}

* Statistically different (ANOVA performed with $95 \%$ of confidence level).

when compared with the conventional hydrogel CL (Etafilcon A). Despite the diversity of proteins observed in Galyfilcon A using SDS-PAGE, this lens is not more prone to protein adsorption than the other silicone hydrogel CL. It must be stressed that the amounts of proteins were estimated using BSA as standard-therefore, the values presented cannot be seen as absolute amounts of proteins.

\subsection{Microbial colonisation}

The values of CFU/ml present in Table 5 indicate the amount of microbial cells able to grow on TSA plates at $37^{\circ} \mathrm{C}$, and are estimates of the extent of the CL's colonisation with viable microbial cells. The results show that Balafilcon A seems to be more prone to microbial colonisation than the other CL, exhibiting an average value of $2.32 \times 10^{6} \mathrm{CFU} / \mathrm{ml}$, which is statistically significant $(p<0.005)$. Microbial colonisation in Galyfilcon A, despite the absence of surface treatment, was similar to those of Lotrafilcon A $(p=0.231)$ and Lotrafilcon B $(p=0.817)$. Concerning the conventional hydrogel CL (Etafilcon A), this lens exhibited a greater amount of viable cells than Galyfilcon A $(p=0.017)$ and Lotrafilcon A $(p=0.00)$.

Table 5

Colony-forming units of worn conventional and silicone hydrogel contact lenses Mann-Whitney $U$ performed with $95 \%$ of confidence level

Etafilcon A

Galyfilcon A

Balafilcon A

Lotrafilcon A

Lotrafilcon B

$9.30 \times 10^{5} \pm 3.49 \times 10^{5 * *}$
$4.08 \times 10^{5} \pm 2.05 \times 10^{5}$
$2.32 \times 10^{6} \pm 1.45 \times 10^{6^{*}}$
$2.30 \times 10^{5} \pm 1.17 \times 10^{5}$
$8.83 \times 10^{5} \pm 7.84 \times 10^{5}$

* Statistically different from all tested lenses.

** Statistically different from Galyfilcon A and Lotrafilcon A.

\section{Discussion}

The present study focuses the effect of silicone hydrogel CL's surface treatment on hydrophobicity, protein adsorption, and microbial colonisation. Due to the important role of hydrophobicity in protein adsorption and microbial colonisation, this property was evaluated in a quantitative way, contrarily to most of the studies [17-19].

In the present study, it was found that the silicone hydrogel CL with surface treatments (Table 2) and the nonsurface-treated CL (Galyfilcon A) present similar degrees of hydrophobicity, meaning that the wetting agent and the application of a surface treatment have a similar effect on the lens hydrophobicity. The conventional hydrogel $\mathrm{CL}$ is hydrophilic, as reported by other studies [17].

SDS-PAGE analysis (Table 3) revealed different protein profiles on the several lens materials. Proteins with molecular weights equivalent to the molecular weights of lactoferrin and lysozyme were the most frequently extracted from all lens materials, probably on account of their abundance in the tear-film [20]. It seems that $14.4 \mathrm{kDa}$ proteins (probably lysozyme) preferentially adsorb onto Etafilcon A CL. This is probably due to the electrostatic attraction between these two entities, since this protein is positively charged at physiological $\mathrm{pH}$, while Etafilcon A is negatively charged. It is well reported that protein adsorption is a phenomenon determined by the lens hydrophobicity and in lesser extent, by the hydrophobicity of the proteins' amino acid residues. This interaction is also influenced by the electrostatic attraction between the lens surface and proteins with opposite charges [21]. Galyfilcon A adsorbed a greater diversity of proteins when compared with all the other lenses, which may be related to the absence of surface treatment and also to its chemical composition. It must be stressed that this type of lens was worn for a longer period than that recommended by the manufacturer (30 days). Nevertheless, neither signs of material degradation nor wearer discomfort were observed (data not shown) during the wearing period.

The fluorescence data (Table 4) revealed that Etafilcon A adsorbs a greater amount of proteins than silicone hydrogel CL. This result has been previously reported by several authors $[9,12,13]$, while specifically studying "in vitro" lysozyme adsorption. Hydrophilic polymers such as Etafilcon A naturally hinder protein adsorption because 
the water must be displaced for protein adsorption to occur, and this process is energetically unfavourable. However, the accentuated dehydration of this lens [22] could allow the interaction with other molecules - in this case, proteins. "In vitro" dehydration studies have revealed that conventional hydrogel CL are more prone to dehydratation than silicone hydrogel CL [23], so, regardless of their hydrophilicity, conventional hydrogel CL adsorb more proteins than the other lenses, even if worn for a shorter period of time (15 days). Concerning Galyfilcon A, it was interesting to conclude that the absence of surface treatment did not lead to an increase in the amount of proteins adsorbed, despite the great diversity of proteins adsorbed. It should be considered the possibility that the variety and amount of proteins adsorbed onto the different CL could have been influenced by the lens material or by the lens care solution used. According to Pritchard et al. [24], the use of ReNu Multiplus has been associated to higher levels of corneal staining when compared to ReNu Multipurpose Solution and Opti-Free Express. The measurement of the corneal staining is a useful tool to determine the impact of a multipurpose system or the impact of their interaction with the lens material on cornea. Despite being out of the aim of the present study, this evaluation was performed. The main finding is that all CL wearers exhibited corneal staining with the exception of one silicone hydrogel lens. As different levels of corneal abrasion may induce different levels in irritation and protein secretion, we believe that the protein levels and profiles observed for each CL may have been influenced either by the multipurpose lens care solution as by their interaction with lens material.

Regarding CFU's analysis, the CL presented different levels of microbial colonisation (Table 5), Balafilcon A being the lens more prone to microbial colonisation. This fact may be related to its hydrophobicity, as it is already well established that microbial adhesion is determined by lens surface hydrophobicity [25], as well as microorganisms, are usually negatively charged. However, hydrophobic interactions are stronger than repulsive forces and tend to attract bacteria near to the surface, leading to their adhesion [26]. Moreover, this lens presents a high electron acceptor capacity, which may possibly enhance adhesion on account of the increase in Lewis acid base interactions with the microbial cells. In a previous report in which "in vitro" adhesion of Staphylococcus epidermidis and Pseudomonas aeruginosa to several silicone hydrogel CL was studied, Balafilcon A was also shown to be more prone to adhesion than the other lenses [25]. In that study, the adhesion assays were performed on unworn contact lenses. However, "in vivo" CL are subjected to the adsorption of tear-film molecules that may influence lens surface's properties [19] and adhesion propensity. Nevertheless, both "in vivo" and "in vitro" studies led to the same conclusion concerning the high susceptibility to microbial colonisation of Balafilcon A. One other factor that may bear influence on this lens's susceptibility to microbial colonisation is its high roughness.
Balafilcon A is rougher than both Lotrafilcon A and Galyfilcon A due to its surface treatment, which presents silicate islands [27]. The surface treatment of this lens based on plasma oxidation is different from those of Lotrafilcon A and Lotrafilcon B, which are made through plasma coating, resulting in a smoother surface with a high refractive index. It was previously demonstrated that microbial adhesion may increase by enhancing roughness [28,29], and for this reason we believe that this surface property may have played an important role in microbial colonisation.

Summarising, all silicone hydrogel CL are hydrophobic and adsorb smaller amounts of proteins than the conventional hydrogel $\mathrm{CL}$, regardless of the presence of surface treatment. Nonetheless, all lenses exhibited the presence of different protein profiles. The possibility of the multipurpose lens care solution or the lens material having an impact on this result should not be excluded, since shifts on ocular irritation may induce different protein secretion. The surface-treated Balafilcon A seems to be more prone to microbial colonisation, which may be related to its greater hydrophobicity and higher electron acceptor capacity. In terms of clinical implications, there are apparently no differences between surface-treated and untreated CL, except for Balafilcon A, since it exhibited a higher amount of colonising microbes.

\section{Acknowledgements}

The authors fully acknowledge the financial support of the Portuguese Foundation for Science and Technology (FCT) through the project POCTI/FCB/44628/2002 and also the grant BD 19679/2004 (FCT).

\section{References}

[1] Holden BA, Sankaridurg P, Jalbert I. Silicone hydrogels-adverse events, the epithelium in extended wear and inflammation and infection in the closed eye. Contact Lens Monthly 2000;219:34-42.

[2] Tighe B, Brennan N, Coles C. Silicone hydrogels-what are they and how should they be used in everyday practice? Contact Lens Monthly 1999;218:31-5.

[3] Bailey CS. Contact lens complications. Optometry June 1999;26-35.

[4] Fleiszig SMJ, Evans DJ. The pathogenesis of bacterial keratitis: studies with Pseudomonas aeruginosa. Clin Exp Optom 2002;85.5: 271-8.

[5] Tighe B, Franklin V. Lens deposition and spoliation. In: The eye in contact lens wear. Butterworth Heinemann; 1985. p. 49-100.

[6] Willcox MDO, Holden BA. Contact lens related corneal infections. Biosci Rep 2001;21:445-61.

[7] Lloyd AW, Faragher RGA, Denyer SP. Ocular biomaterials and implants. Biomaterials 2001;22:769-85.

[8] Franklin V, Horne A, Jones L, Tighe B. Early deposition trends on group I (Polymacon and Tetrafilcon A) and group III (Bufilcon A) materials. CLAO J 1991;17:244-8.

[9] Jones L, Senchyna M, Glasier MA, Schickler J, Forbes I, Louie D, et al. Lysozyme and lipid deposition on silicone hydrogel contact lens contact lens material. Eye Contact Lens 2003;29:S75-9. 
[10] Keith EO, Boltz M, Gadh R, Ghorsriz R, Mangatt D, Janoff LE. Adhesion of tear proteins to contact lens and vials. Biotechnol Appl Biochem 2001;34:5-12.

[11] Moradi O, Modarress H, Noroozi M. Experimental study of albumin and lysozyme adsorption onto acrylic acid (AA) and 2-hydroxyethyl methacrylate (HEMA) surfaces. J Colloid Interf Sci 2004;271:16-9.

[12] Subbaraman LN. Lysozyme deposition studies on silicone hydrogel contact lens materials. Master Thesis. University of Waterloo, Ontario, 2005. p. 75-6.

[13] Zhang S, Borazjani RN, Salamone JC, Ahearn DG, Crow Jr SA, Pierce GE. In vitro deposition of lysozyme on etafilcon A and balafilcon A hydrogel contact lenses: effects on adhesion and survival of Pseudomonas aeruginosa and Staphylococcus aureus. Contact Lens Anterior Eye 2005;28:113-9.

[14] Ladokhin AS. In: Meyers RA, editor. Fluorescence spectroscopy in peptide and protein analysis. Chichester: John Wiley \& Sons Ltd.; 2000. p. 5762-79.

[15] Keith D, Hong B, Christensen M. A novel procedure for the extraction of protein deposits from soft hydrophilic contact lenses for analysis. Curr Eye Res 1997;16:503-10.

[16] van Oss CJ, Giese RF. The hydrophilicity and hydrophobicity clay minerals. Clay Miner 1995;43:474-7.

[17] Bruinsma GM, van der Mei HC, Busscher HJ. Bacterial adhesion to surface hydrophilic and hydrophobic contact lenses. Biomaterials 2001;22:3217-24.

[18] Cheng L, Muller SJ, Radke CJ. Wettability of silicone-hydrogel contact lenses in the presence of tear-film components. Curr Eye Res 2004;28:93-108.

[19] Shirafkan A, Woodward EG, Port MJA, Hull CC. Surface wettability and hydrophilicity of soft contact lens materials, before and after wear. Ophthal Physiol Opt 1995;15:529-32.
[20] Sariri R. Protein interaction with hydrogel contact lenses. J Appl Biomater Biomech 2004;2:1-19.

[21] Elbert DL, Hubbel JA. Surface treatments of polymers for biocompatibility. Annu Rev Mater Sci 1996;26:365-94.

[22] Tranoudis I, Efron N. Water properties of soft contact lens materials. Contact Lens Anterior Eye 2004;27:193-208.

[23] Jones L, May C, Nazar L, Simpson T. In vitro evaluation of the dehydratation characteristics of silicone hydrogel and conventional hydrogel contact lens materials. Contact Lens Anterior Eye 2002;25:147-56.

[24] Pritchard N, Young G, Coleman S, Hunt C. Subjective and objective measures of corneal staining related to multipurpose systems. Contact Lens Anterior Eye 2003;26:3-9.

[25] Henriques M, Sousa C, Lira M, Real Oliveira MECD, Oliveira R, Azeredo J. Adhesion of Pseudomonas aeruginosa and Staphylococcus epidermidis to silicone hydrogel contact lens. Optom Vis Sci 2005;82:446-50.

[26] Gristina AG, Naylor PT. Implant-associated infection. In: Ratner BD, Hoffman AS, Schoen FJ, Lemons JE, editors. Biomaterials sciencean introduction to materials in medicine. Academic Press; 1996. p. 205-14.

[27] González-Méijome JM, López-Alemany A, Almeida JB, Parafita MA, Refojo MF. Microscopic observation of unworn siloxane-hydrogel soft contact lenses by atomic force microscopy. Appl Biomater 2006;76B:412-8.

[28] Gallardo-Moreno AM, González-Martin ML, Bruque JM, Pérez-Giraldo C. The adhesion of Candida parapsilosis to glass and silicone as a function of hydrophobicity, roughness and cell morphology. Colloids Surf A: Physicochem Eng Aspects 2004;249:99-103.

[29] Packham DE. Surface energy, topography and adhesion. Int J Adhes Adhes 2003;23:437-48. 